\title{
Effects of work function and electron affinity on the performance of carrier-selective contacts in silicon solar cells using $\mathrm{ZnSn}_{\mathrm{x}} \mathrm{Ge}_{1-\mathrm{x}} \mathrm{N}_{2}$ as a case study
}

\author{
Davi Fébba, Graduate Student Member, IEEE, Vincent Paratte, Luca Antognini, Julie Dréon, Julien Hurni, \\ Jonathan Thomet, Rero Rubinger, Edson Bortoni, Christophe Ballif, Mathieu Boccard
}

\begin{abstract}
This work reports the electrical characterization of $\mathbf{Z n S n}_{\mathbf{x}} \mathbf{G e}_{1-\mathbf{x}} \mathbf{N}_{\mathbf{2}}$ (ZTGN) layers $(10 \%<x<90 \%)$ deposited on glass by combinatorial sputtering and further assesses the performance of silicon heterojunction (SHJ) solar cells featuring them as electron-selective contacts. Bandgap, dark conductivity, and the activation energy of the latter were found to significantly change between $\mathrm{Sn}$ and Ge-rich samples. When applying ZTGN layers as electron-selective contacts for SHJ solar cells, poor solar-cell performance was observed, with surprisingly similar results despite changes in material properties. From analysis and modelling of the current-voltage characteristics using several device structures, we show that the work function of the electronselective contact lies around $4.35 \mathrm{eV}$ for all investigated $\mathrm{Sn}$ and Ge contents, which is too high to form an excellent electronselective contact. By comparing different solar-cell architectures, we could further identify that the Ge-rich layer imposes an additional barrier to electron extraction, independently of its poor selectivity, due to its low conductivity. Doping of Ge-rich ZTGN appears thus as the most relevant approach to build efficient devices with a ZTGN contact layer.
\end{abstract}

Index Terms- $\mathrm{ZnSnN}_{2}, \mathrm{ZnGeN}_{2}$, carrier-selective contact, silicon heterojunction, nitrides, solar cells, band structure.

\section{INTRODUCTION}

The search for new Earth-abundant and low-cost materials for PV applications has led to a recent interest in II-IV-N 2 semiconductors. Among these, $\mathrm{Zn}_{\mathrm{x}} \mathrm{Sn}_{1-\mathrm{x}} \mathrm{N}_{2}$ is an interesting candidate due to a tunable direct bandgap depending on stoichiometry $(0<\mathrm{x}<1)$, with values ranging from 0.7 to $2.8 \mathrm{eV} \mathrm{[1],} \mathrm{and} \mathrm{strong} \mathrm{optical} \mathrm{absorption} \mathrm{in} \mathrm{the} \mathrm{solar} \mathrm{spectrum}$ [2]. Alloying $\mathrm{ZnSnN}_{2}$ with $\mathrm{ZnGeN}_{2}$, another direct bandgap semiconductor but with wider energy gaps in the range 2.7$3.4 \mathrm{eV}$ [3], results in $\mathrm{Zn}\left(\mathrm{Sn}_{\mathrm{x}} \mathrm{Ge}_{1-\mathrm{x}}\right) \mathrm{N}_{2}$ (ZTGN) compounds that can extend the bandgap range further up, while maintaining a II/IV ratio of 1 [4].

D. F. was sponsored by the National Council for Scientific and Technological Development $(\mathrm{CNPq}-\mathrm{Brazil})$ and by the Federal Commission for Scholarships for Foreign Students (FCS - Switzerland). This work was supported by the Swiss National Science Foundation under Ambizione Energy Grant ICONS (PZENP2_173627).

The authors are with the Photovoltaics and Thin Film Electronics Laboratory, Institute of Microengineering, École Polytechnique Fédérale de Lausanne (EPFL), CH-2000 Neuchâtel, Switzerland (e-mail: davifebba@uol.com.br). R. Rubinger and E. Bortoni are with the Universidade Federal de Itajubá (UNIFEI), 37500-903 Itajubá, Brazil. DOI: 10.1109/JPHOTOV.2021.3109585. (C) 2021 IEEE. Personal use of this material is permitted. Permission from IEEE must be obtained for all other uses, in any current or future media including reprinting/republishing this material for advertising or promotional purposes, creating new collective works, for resale or redistribution to servers or lists, or reuse of any copyrighted component of this work in other works.
Moreover, given the width of its bandgap tunability, ZTGN can be applied either as a PV absorber $(x=1$, corresponding to ZTN, for example), or as a contacting material for heterojunction solar cells when the Ge concentration increases and ZTGN shows wide bandgaps. In the latter case, it is interesting to note that this material could, at the same time, be used as absorber and contact layer in the same structure, by only changing its stoichiometry.

As a new system of materials, research on ZTGN is still in its early stages. The first work reporting its synthesis and structural characterization dates back to 2012 [5]. This was motivated by the bandgap tunability of $\operatorname{In}_{\mathrm{x}} \mathrm{Ga}_{1-\mathrm{x}} \mathrm{N}$ alloys, and proposed the replacement of the group III by group II ( $\mathrm{Zn}$ ) and IV (Sn, Ge) elements to improve raw-material availability for large-scale PV applications.

Bandgap tunability of ZTGN was experimentally demonstrated in [6], which reported an increase in bandgap from $2 \mathrm{eV}$ to $3.1 \mathrm{eV}$, mostly due to a shift in the conduction band to higher energies as the Ge content increased. Since then, other works reported bandgaps in the range 2.1-3.01 eV [7] and $2.2-2.7 \mathrm{eV}$ [8]. Moreover, ZTGN presents also a wide range of conductivity (n-type) depending on composition, with an exponential increase in conductivity as the $\mathrm{Sn}$ content increases, along with a decrease of its dark conductivity activation energy [7], [8].

Yet, the accomplishment of PV devices with this family of semiconductors remains a largely unexplored research topic, with few works reporting PV devices with ZTN as absorber [9]-[11] and only one studying ZTN as electron-selective contact for silicon solar cells [12]. Nevertheless, the wide ranges of values for bandgap and conductivity of ZTGN alloys makes them interesting materials to study as electronselective contact. Historically, SHJ cells have been used as test platform for evaluating novel carrier-selective contacts since it combines a nearly perfect absorber (crystalline silicon), interlayers inherently providing excellent passivation without any selectivity (intrinsic amorphous silicon), and nearly perfect carrier-selective layers (doped silicon layers) [13], [14].

In this context, we fabricated by combinatorial sputtering ZTGN layers of varying $\mathrm{Zn}$, Sn and Ge content to assess their effectiveness as electron-selective contacts in SHJ solar cells. We characterized their optical and electrical properties and analyzed several SHJ solar cells with different architectures featuring these layers as electron-selective contact. By modelling the current-voltage characteristics of these solar cells, 
we draw the band alignment of $\mathrm{Zn}\left(\mathrm{Sn}_{\mathrm{x}} \mathrm{Ge}_{1-\mathrm{x}}\right) \mathrm{N}_{2}$ with silicon for various $\mathrm{x}$ values. Based on these results, we discuss the impact of work function and electron affinity on solar cell performance and evidence two different possible causes for poor device performance.

\section{LAYERS ON GLASS}

\section{A. Fabrication and characterization methods}

ZTGN layers were initially deposited on glass for material characterization through a combinatorial co-sputtering approach to assess a wide range of composition in a single deposition run. The chamber was first evacuated to a pressure of $2.6 \times 10^{-7}$ mbar, and then a constant flow of $17.5 \mathrm{sccm}$ $\mathrm{N}_{2}$ was introduced in the chamber to reach $1.3 \times 10^{-2}$ mbar. The power of RF generators on the Sn (99.995\% purity) and Ge (99.999\% purity) metallic targets (100-mm diameter) was adjusted at 59 and $100 \mathrm{~W}$, respectively, while the $\mathrm{Zn}$ target (99.98\% purity) was kept at a DC power of $45 \mathrm{~W}$, leading to an estimated $50 \% \mathrm{Zn} / 25 \% \mathrm{Sn} / 25 \%$ Ge composition at the central position of the substrate (assessed from deposition rate from each target individually). The substrate was not rotated during deposition, which created a gradient in composition. Deposition lasted 30 minutes, following 8 minutes of presputtering.

The resulting combinatorial library, with $0.49 \mathrm{~cm}^{2}$ samples, was subjected to ellipsometry measurements over the range $0.6-6 \mathrm{eV}$ at an angle of $70^{\circ}$ using a Horiba Jobin Yvon ellipsometer. The experimental data was fitted to the TaucLorentz model through the DeltaPsi2 software to extract thickness and optical bandgap $\left(\mathrm{E}_{04}\right.$, the energy at which the absorption coefficient falls below $1 \times 10^{4} \mathrm{~cm}^{-1}$ ).

For dark conductivity measurements, $100 \mathrm{~nm}$ thick and $8 \mathrm{~mm}$ long Al pads, $1 \mathrm{~mm}$ spaced, were evaporated on the samples which were then annealed at $210^{\circ} \mathrm{C}$ for $30 \mathrm{~min}$. The samples were placed on a temperature-controlled chuck inside a vacuum chamber, with $\mathrm{N}_{2}$ atmosphere at 1 mbar, for temperature-dependent dark conductivity measurements. For these measurements, the samples were annealed at $180^{\circ} \mathrm{C}$ for 1.5 hours, followed by a slow temperature decrease down to $30^{\circ} \mathrm{C}$ (5 hours in total) during which conductivity was monitored. Conductivity at $20^{\circ} \mathrm{C}$ and activation energy were both extracted from Arrhenius plots, by fitting conductivity data during the temperature ramp down to $\sigma(T)=$ $\sigma_{0} \exp \left(-E_{A} / k T\right)$, where $k, E_{A}$ and $\sigma_{0}$ are the Boltzmann constant, activation energy and an exponential factor which corresponds to the conductivity as $T \rightarrow \infty$, respectively.

\section{B. Optoelectronic properties}

Fig. 1 shows a picture of the resulting ZTGN combinatorial library, together with the Arrhenius conductivity plots, thickness and $\mathrm{E}_{04}$ for positions 1 to 5. Thickness was around $100 \mathrm{~nm}$, with the Sn-rich side appearing slightly thicker. Also, $\mathrm{E}_{04}$ shifted to higher energies as the Ge content increased, which confirmed the findings obtained in previous studies [6][8], where an increase in bandgap was seen for samples with higher Ge content.

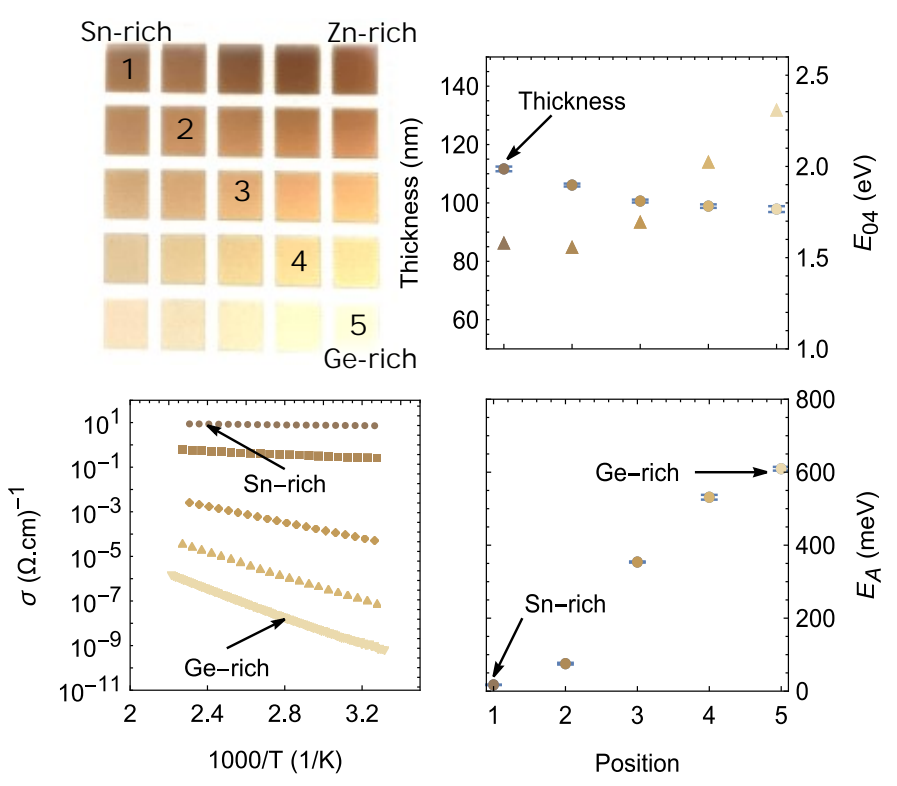

Fig. 1. Photograph of the combinatorial ZTGN samples deposited on glass (top left), and material properties against position on the combinatorial library. Position 1 and 5 are Sn and Ge-rich, respectively.

Conductivity and activation energy were found to strongly change between $\mathrm{Sn}$ and Ge-rich samples, going from $7 \mathrm{~S} \mathrm{~cm}^{-1}$ and $17 \mathrm{meV}$ at the Sn-rich position, to $2.5 \times 10^{-10} \mathrm{~S} \mathrm{~cm}^{-1}$ and $610 \mathrm{meV}$ at the Ge-rich position. These findings corroborated previous works [7], [8], where a strong decrease in resistivity was observed when the Ge content increased, along with an increase in activation energy. Moreover, as observed in [7], although the Ge-rich sample showed an exponential increase in conductivity as the temperature increased, the Snrich sample showed only a small and linear increase. This corresponds to an intermediate behavior between semiconductorlike and metal-like, suggesting a (close-to-)degenerate doping. This explains the slightly higher $\mathrm{E}_{04}$ value for position 1 than position 2 in spite of a higher Sn content.

Thus, increasing the Ge content resulted in more insulating samples showing an increased bandgap and activation energy. This trend was attributed in [8] to the higher electronegativity of $\mathrm{Ge}$ than of Sn, making it a poorer electron donor, ultimately leading to a reduced density of thermally activated carriers in the conduction band.

\section{SOLAR CELLS}

\section{A. Fabrication and characterization methods}

Front-junction SHJ solar cells were fabricated through standard protocols on chemically etched c-Si wafers. Both n- and p-type float zone (FZ) wafers were used, with thickness and resistivity around $200 \mu \mathrm{m}$ and $2 \Omega \mathrm{cm}$, respectively. Intrinsic and doped amorphous silicon (a-Si:H) were deposited by PECVD, and ITO was sputtered on top of the Si wafer with a metallic grid, defining $0.49 \mathrm{~cm}^{2}$ solar cells as sketched in Fig. 2. The backside of each wafer was covered with ITO/Ag by magnetron sputtering, and silver paste contacts were then applied on the front of each cell to enable contacting. Also, 
a)

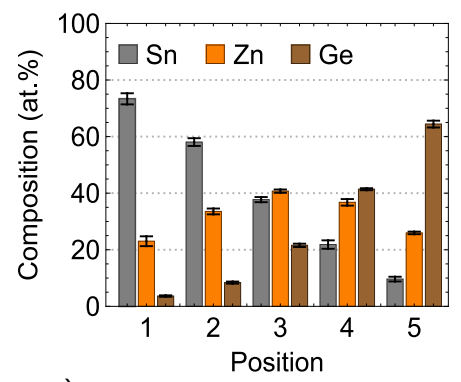

b)

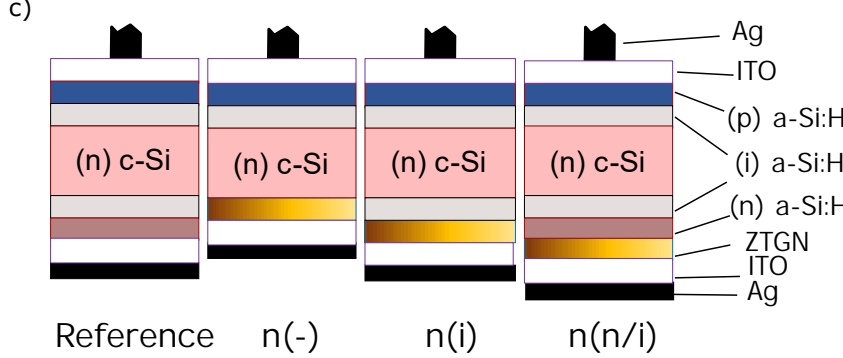

Fig. 2. a) Estimated composition (at.\%) of each solar cell on 5 positions on the silicon wafer, shown in b), where $\mathrm{Zn}, \mathrm{Sn}$ and $\mathrm{Ge}$ denote the approximate positions of the sputtering guns against the wafer; c) architectures of the investigated SHJ solar cells with ZTGN, and the corresponding reference cell.

as reference, SHJ solar cells were fabricated with standard front-junction architecture on $\mathrm{n}$ and $\mathrm{p}$-type $\mathrm{Si}$.

Fig. 2 also shows the results of Energy Dispersive Spectroscopy (EDS), which was carried out on ZTGN layers grown on a Double Sided Polished (DSP) p-Si wafer to investigate the $\mathrm{Zn}, \mathrm{Sn}$ and Ge composition at five positions corresponding to the ones of the studied solar cells. The sputtering conditions were the same as for solar cells, but deposition time was increased to 1 hour, targeting $200 \mathrm{~nm}$ thick layers at the center of the silicon wafer.

The resulting solar cells had a $\mathrm{Sn} /(\mathrm{Sn}+\mathrm{Ge})$ ratio ranging from $(90 \pm 1) \%$ (position 1) to $(10 \pm 1) \%$ (position 5), which shows that the combinatorial approach was effective at exploring a wide range of cation composition. The uncertainty of the atomic percentage of each element was evaluated as the $95 \%$ confidence interval for the mean, estimated from measurements over several areas on different spots at each position.

Furthermore, as sketched in Fig. 2, the ZTGN layer was placed either as passivating electron-selective contact (replacing both the intrinsic and (n) a-Si:H films), or simply as electron-selective contacts on top of an (i) a-Si:H layer, or even as spectator between the (n) a-Si:H layer and ITO, according to the methodology presented in [12]. The sputtering parameters were the same as for samples deposited on glass, except that deposition time was reduced to 8 minutes, after a 2minute pre-sputtering step, targeting layers $20 \mathrm{~nm}$ thick at the central position of the silicon wafer, which was confirmed by ellipsometry.

Five solar cells from each wafer were subjected to IV characterization using a custom-made solar simulator developed at PV-LAB. For that, solar cells were covered with a mask of $0.49 \mathrm{~cm}^{2}$ and positioned on a temperature-controlled vacuum
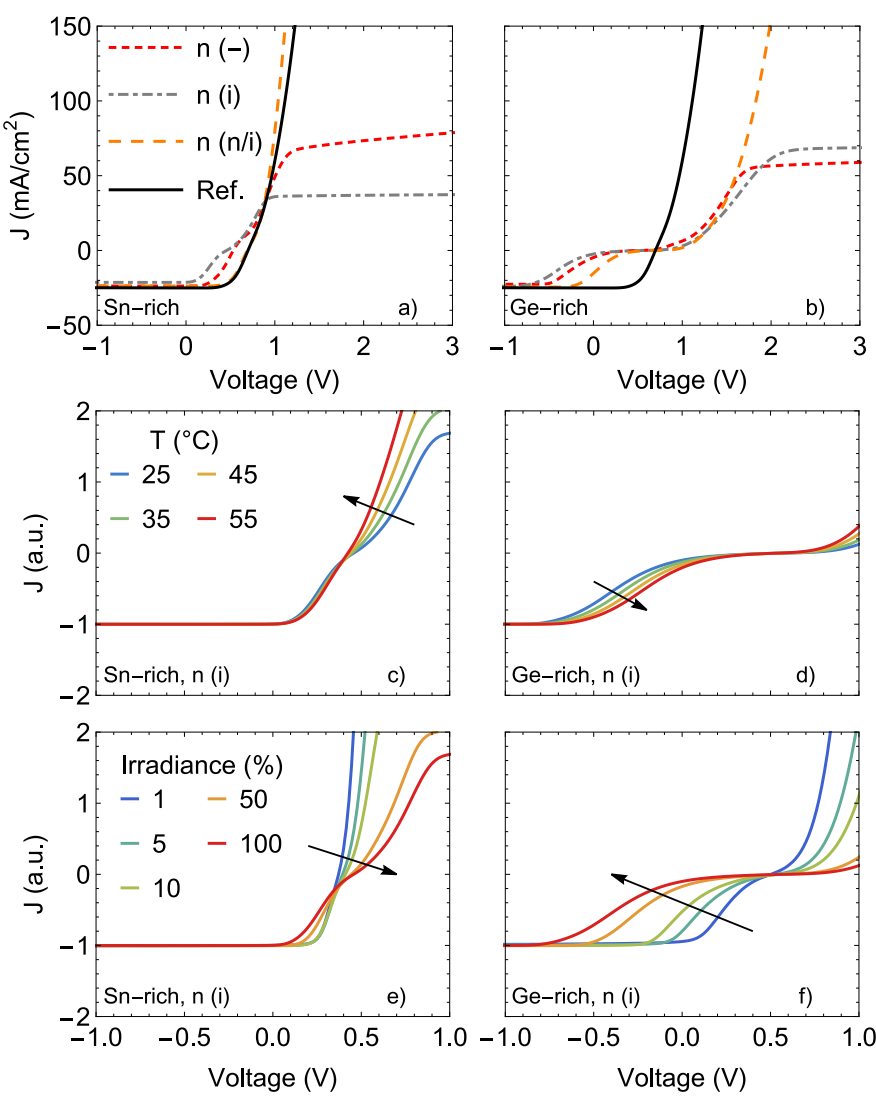

Fig. 3. JV curves at STC with a) Sn and b) Ge-rich ZTGN layers at STC (a standard SHJ solar cell JV curve is also shown as reference). c) and d) show the temperature effects on the JV characteristics of the $\mathrm{Sn}$ and Ge-rich solar cells, respectively, from architecture "n(i)", while e) and f) show the irradiance effects on the same cells. JV curves were normalized to $J_{p h}$ in c), d), e) and f), and arrows denote increasing temperature and irradiance.

chuck at $25^{\circ} \mathrm{C}$, kept stable by a PID controller, a PT100 temperature sensor and peltier modules. The JV characteristics were then measured with the 4-wires approach by a Keithley 2601 Source Measure Unit (SMU) at five different levels of irradiance: $1,5,10,50$ and $100 \%$ of $1000 \mathrm{~W} \mathrm{~cm}^{-2}$, provided by LED and halogen lamps.

\section{B. Device architecture: impacts on JV characteristics}

Fig. 3 a) and b) show the JV characteristics (STC) of solar cells with $\mathrm{Sn}$ and Ge-rich ZTGN composition, after annealing for $30 \mathrm{~min}$ at $210^{\circ} \mathrm{C}$, for the three architectures tested on $\mathrm{n}-\mathrm{Si}$. The other three solar cells, at positions $2-4$, showed a behavior similar to that of the Sn-rich one, and therefore are not shown.

Common features were observed when ZTGN was used as selective contact, i.e. structures $\mathrm{n}(-)$ and $\mathrm{n}(\mathrm{i})$, regardless of composition: two steps in current (one around $\mathrm{V}_{\mathrm{oc}}$ and another at high bias) and a saturation behavior in forward bias (strict when a-Si:H was present, but with a slight current drift as voltage increases in absence of the intrinsic a-Si:H).

Inserting an (n) a-Si:H before the ZTGN layer, which relaxes the electron-selectivity requirements for the ZTGN layer, brings a clear difference between the two ZTGN compositions: in the Sn-rich case (Fig. 3 a)), the s-shape around $V_{\text {oc }}$ and the saturation were suppressed, resulting in an IV curve similar to 
(and even slightly better than) the reference cell. Conversely, for the Ge-rich sample, the rectification was suppressed, but the strong s-shape around $\mathrm{V}_{\mathrm{oc}}$ was maintained. These results suggest that both layers lack electron selectivity since device performance benefited from the addition of the (n) a-Si:H layer, but the Ge-rich layer imposes an additional barrier to the electron flow compared to the Sn-rich case. These two different sources of barrier to electron extraction are further discussed in the next sections.

Fig. $3 \mathrm{c}$ ) to $\mathrm{f}$ ) further show irradiance- and temperaturedependent IV measurements of the devices using an intrinsic passivation layer under the ZTGN film (no (n) a-Si:H layer), to further investigate the S-shape observed at STC. As reported in [15], if transport through a barrier is the limiting factor, decreasing the temperature reduces the amount of charge carriers crossing the barrier, leading to an accumulation of carriers near the interface, reducing the collection efficiency and causing an early onset of the s-shape curve. Accordingly, the s-shapes around $\mathrm{V}_{\mathrm{oc}}$ were reduced at high temperatures, enhancing the fill factor, as shown in Fig. 3 c) and d). For the Ge-rich case, a prominent S-shape remained, suggesting that a barrier is still present. This can be linked to the high activation energy in the ZTGN layer itself $(>600 \mathrm{mV})$ for which a measurement temperature of $55^{\circ} \mathrm{C}$ is not high enough.

Furthermore, when increasing irradiance, a higher density of photogenerated carriers will be generated, causing also a carrier accumulation at the barrier. This induces a higher band bending and therefore a higher barrier to be overcome, resulting in an earlier onset of the s-shaped curve. Accordingly, Fig. 3 e) and f) shows that reducing the light intensity while keeping the temperature at $25^{\circ} \mathrm{C}$ reduced the s-shapes and its voltage onsets. Overall, these results suggest that a thermionic emission barrier is limiting electron extraction in both cases, though more severely for the Ge-rich case.

\section{Exchange current density modeling}

To further investigate the limiting mechanisms behind these JV characteristics, we fitted temperature-dependent JV measurements for solar cells from positions 1 to 5 using the architecture "n (i)". We used a recent model, presented in [16], that describes the JV characteristics of a contact-limited solar cell based on the equilibrium exchange currents of electrons and holes at each contact. According to this model, the JV characteristics of a contact-limited solar cell can be described by

$$
\begin{aligned}
J(V) & =-\left(J_{L}+j_{0 n}^{\alpha}+j_{0 p}^{\beta}\right)+\frac{J_{L}+j_{0 n}^{\alpha}+J_{0 n}^{\beta}}{1+\frac{J_{0 n}^{\beta}}{j_{0 n}^{\alpha}} e^{-\left(V-J R_{s}\right) / V_{T}}} \\
& +\frac{J_{L}+j_{0 p}^{\beta}+J_{0 p}^{\alpha}}{1+\frac{J_{0 p}^{\alpha}}{j_{0 p}^{\beta}} e^{-\left(V-J R_{s}\right) / V_{T}}}
\end{aligned}
$$

where $V_{T}$ is the thermal voltage; $J_{0 p}^{\alpha}$ and $J_{0 n}^{\beta}$ denote majority hole and electron currents to the $\alpha$ and $\beta$ contacts, while $j_{0 n}^{\alpha}$ and $j_{0 p}^{\beta}$ represent the minority electron and hole currents to the $\alpha$ and $\beta$ contacts, which represent the hole and electron
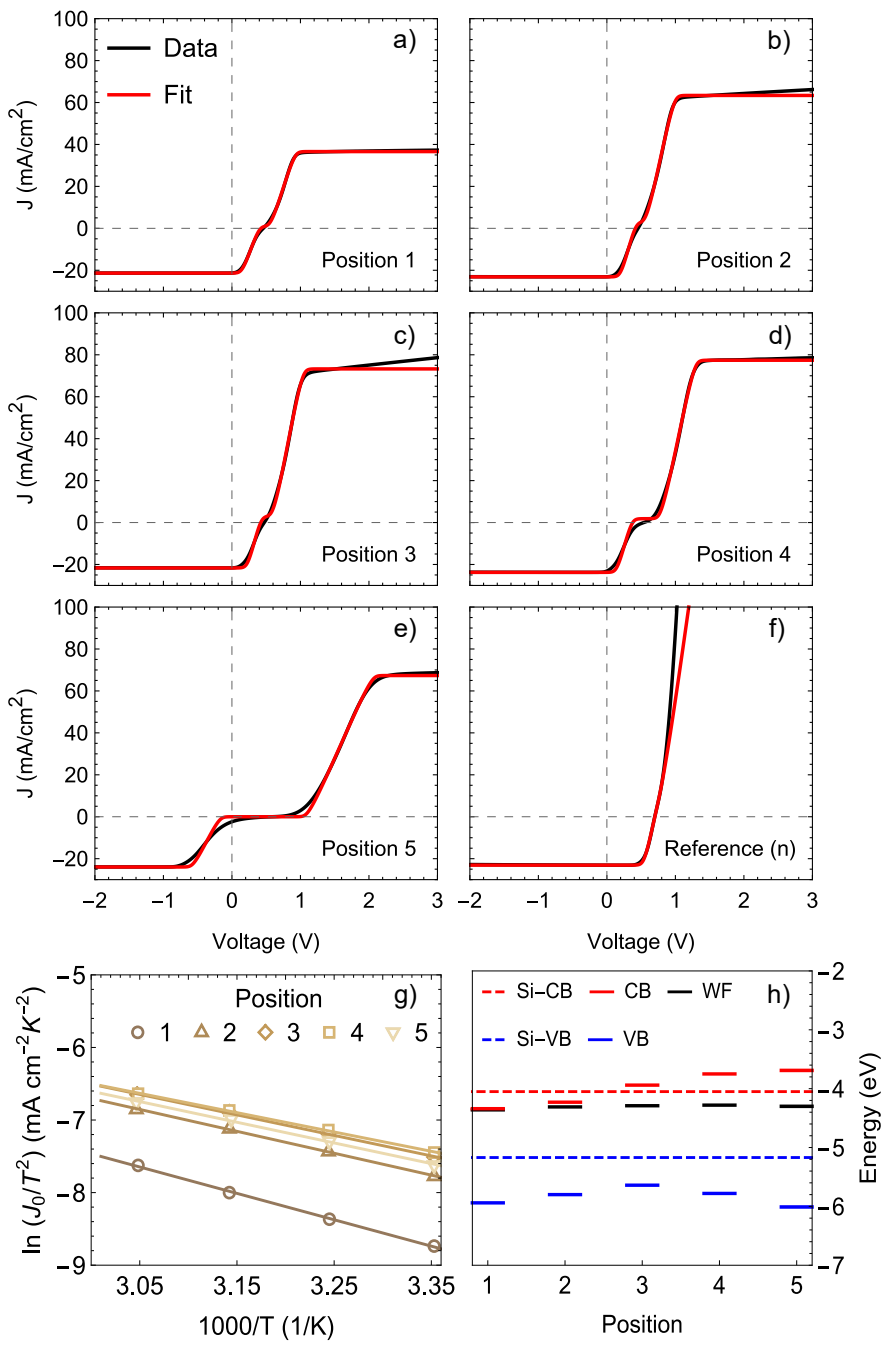

Fig. 4. Top: JV data and the resulting fittings to the model given in (1) for solar cells at positions 1 to 5 and for a reference cell, at STC. Bottom: Richardson plot of the electron current to the electron contact for solar cells at positions 1 to 5 , extracted from the fittings shown in the top box, between 25 and $55^{\circ} \mathrm{C}$ at $1000 \mathrm{~W} \mathrm{~m}^{-2}$. A suggested band alignment between $\mathrm{c}-\mathrm{Si}$ and ZTGN is also presented.

contacts, respectively. Moreover, a series resistance term was added to account for ohmic losses, as in [17].

The resulting IV-T curves were then fitted to (1) between $-0.2 \mathrm{~V}$ and $2 \mathrm{~V}$, by a self-adaptive Differential Evolution algorithm, introduced in [18] and already proven robust against measurement noise [19]. This method is extensively described in the literature, and its details can be found in the references provided here.

Fig. 4 shows the JV data and the corresponding fittings for solar cells at positions 1 to 5 (a reference cell JV data was included for comparison), at STC. The leveling around $\mathrm{V}_{\mathrm{oc}}$ and the saturation in forward bias are well reproduced with this model, resulting in the $\mathrm{J}_{0}$ values shown in Table I.

For positions 1 to 3 , the JV data could be acceptably reproduced with similar, realistic values for the currents on the p-aSi side $\left(j_{0 n}^{p-a S i}\right.$ and $\left.J_{0 p}^{p-a S i}\right)$. For positions 4 and 5 , acceptable fitting is also obtained, but a strong decrease in the electron current to the hole contact was required to 
TABLE I

EQUILIBRIUM-EXCHANGE-CURRENT-DENSITY, SERIES-RESISTANCE, AND WORK-FUNCTION VALUES EXTRACTED FROM THE FITS SHOWN IN FIG. 4

\begin{tabular}{|c|c|c|c|c|c|c|}
\hline & $j_{0 n}^{p-a S i}$ & $\begin{array}{l}J_{0 p}^{p-a S i} \\
\quad(m A)\end{array}$ & $\begin{array}{l}J_{0 n}^{Z T G N} \\
\left.\mathrm{~cm}^{2}\right)\end{array}$ & $j_{0 p}^{Z T G N}$ & $\begin{array}{c}R_{s} \\
\left(\Omega \cdot \mathrm{cm}^{2}\right)\end{array}$ & $\begin{array}{l}\text { WF } \\
(\mathrm{eV})\end{array}$ \\
\hline Position 1 & $7.02 \mathrm{E}-10$ & 0.96 & 14.28 & $1.35 \mathrm{E}-06$ & 7.15 & $4.36 \pm 0.03$ \\
\hline Position 2 & $1.67 \mathrm{E}-09$ & 2.86 & 37.37 & $2.13 \mathrm{E}-06$ & 5.08 & $4.31 \pm 0.02$ \\
\hline Position 3 & $6.11 \mathrm{E}-10$ & 2.91 & 48.69 & $1.65 \mathrm{E}-06$ & 4.58 & $4.29 \pm 0.02$ \\
\hline Position 4 & $4.22 \mathrm{E}-13$ & 1.83 & 51.80 & $9.71 \mathrm{E}-06$ & 5.13 & $4.28 \pm 0.02$ \\
\hline Position 5 & $9.89 \mathrm{E}-19$ & $3.29 \mathrm{E}-13$ & 43.51 & $2.05 \mathrm{E}-09$ & 12.36 & $4.30 \pm 0.04$ \\
\hline Reference (n) & $2.09 \mathrm{E}-11$ & 6.58 & $2.04 \mathrm{E}+06$ & $4.00 \mathrm{E}-12$ & 4.04 & - \\
\hline Position 5 - S & $3.72 \mathrm{E}-11$ & 0.10 & 43.60 & $1.94 \mathrm{E}-06$ & 8.44 & - \\
\hline
\end{tabular}

match the experimental data. There is no physical reason for this decrease since the hole contact is the same for all positions. This suggests that the strong S-shape around opencircuit-voltage of these two samples cannot be modelled with this exchange-current density formalism. As will be discussed in more details in the last section, this is indicative of a conductivity issue [20] for electron extraction, that is unrelated to the selective extraction of charges from the silicon absorber. Nevertheless, the fitted values for the electron current at the ZTGN contact $\left(J_{0 n}^{Z T G N}\right)$ were not deviating significantly from the values obtained at the other positions, since this current is indeed mostly governed by the saturation current density in forward bias, and therefore the results were kept in this work. Conversely, the hole current is mostly governed by the $\mathrm{V}_{\mathrm{oc}}$ value, which is not well defined due to the very flat portion of the curve in this range.

The hole currents on the ZTGN side $\left(j_{0 p}^{Z T G N}\right)$ did not show major changes between positions 1 and 3 , indicative of similar (poor) passivation for all these cases, with a marginal improvement for positions 4 and 5-these should however be taken with caution in view of the questionable validity of the fits as discussed above. Conversely, some improvement was seen for the electron current to the ZTGN contact (which can be visualized on Fig. 4 by an increase of the value at which forward-bias current saturates). Using the formalism of [21], the contact carrier selectivity can be defined by the ratio of the majority to minority carriers to a single contact (so $J_{0 n}^{Z T G N} / j_{0 p}^{Z T G N}$ and $J_{0 p}^{p-a S i} / j_{0 n}^{p-a S i}$ ). Therefore, as expected the electron contact stack (ZTGN) is around 2 orders of magnitude less selective than the hole contact. Conversely, the carrier selectivity of the device can be defined as the ratio $J_{0 n}^{Z T G N} / j_{0 n}^{p-a S i}$ for electrons, and $J_{0 p}^{p-a S i} / j_{0 p}^{Z T G N}$ for holes, respectively. Thus, holes are the limiting carriers in these solar cells, since ZTGN could not prevent a strong hole current to the electron contact, therefore not providing enough electron selectivity for a good cell performance.

Furthermore, considering that transport of electrons to the ZTGN contact is limited by thermionic emission over the barrier at the ZTGN / n-Si interface, the electron exchange currents at the ZTGN contact are expected to follow

$$
J_{0 n}^{Z T G N}=A^{*} T^{2} e^{-\phi / k T}
$$

where $A^{*}$ is the effective Richardson constant and $\phi$ is the barrier height, i.e. the difference between the n-Si electron affinity and the ZTGN work function.
As recently shown in [22], if the thermionic emission hypothesis holds, fitting IV-T to (1) is an effective way to estimate the work function at the contact. Therefore, we plotted in Fig. $4 \mathrm{~g}$ ) the natural logarithm of $J_{0 n}^{Z T G N} / T^{2}$ against $1 / T$ (Richardson plot), and extracted the slope of the resulting trendline to estimate the effective work function of the electron contact for all compositions. (The fittings at $35^{\circ} \mathrm{C}, 45^{\circ} \mathrm{C}$ and $55^{\circ} \mathrm{C}$ are not shown but yielded similar fitting quality to the $25^{\circ} \mathrm{C}$ case).

These extracted work function values (shown in Table I) shuffle around $4.3 \mathrm{eV}$, with only a marginal decrease when going from Sn-rich to Ge-rich. This corresponds to a Fermilevel position $0.25 \mathrm{eV}$ below the conduction band of silicon (and $0.3 \mathrm{eV}$ away from mid-gap). This renders the ZTGN layers electron selective, although not sufficiently to form a good contact as evidenced by the low obtained $\mathrm{V}_{\mathrm{oc}}$ below $600 \mathrm{mV}$, as discussed in next section.

Combining this calculated work function with the activation energy and optical bandgap measurements shown in Fig. 1, Fig. $4 \mathrm{~h}$ ) shows the reconstructed band structure of the different ZTGN alloys, and in particular the alignment with the $\mathrm{c}-\mathrm{Si}$ bands. A shift of the conduction band minimum to higher energies (closer to the vacuum-level) and little movement of the valence band maximum is seen, consistently with earlier reports based on X-ray spectroscopy [6]. The relatively constant work function across variable compositions in spite of moving conduction and valence bands can be put in parallel with the InGaN alloy case. For this alloy, a similar Fermi-level position around $4.6 \mathrm{eV}$ below the vacuum energy was obtained upon introducing defects, independently from the alloy composition. This resulted in defective high-In-content alloys being highly conductive [23]. A similar phenomenon is indeed likely to be at play in the ZTGN family, and the samples fabricated via reactive sputtering are likely to be inherently defective. Going further, inferring from the universal alignment of hydrogen levels in semiconductors and insulators [24], it is tempting to generalize these observations, and postulate that intrinsically conductive semiconductors (which necessarily owes their conductivity from intrinsic defects) are unlikely to perform efficiently as electron-selective contacts, since their intrinsic conductivity is inextricably linked with a too high electron affinity for this role. This would make doping a necessity to build efficient electron-selective contacts which do not rely on an elaborate combination with a low-workfunction metal electrode (as in the $\mathrm{TiO}_{2} / \mathrm{Al}$ [25] or $\mathrm{ZnO} / \mathrm{Al}$ [26] cases to name a few).

\section{Device architecture: effect of the wafer type}

We now discuss the influence of the solar cell architecture, i.e. wafer type, with the presence or not of an (i)a-Si:H passivation layer, and of an (n)a-Si:H layer. In addition to the three architectures discussed previously and shown on Fig. 2, two additional architectures were compared, using a p-type silicon wafer, as shown on Fig. 5. A front-junction configuration was used to avoid collection issues, and the (i)a$\mathrm{Si}: \mathrm{H}$ passivation layer was used or omitted.

For cells featuring only the ZTGN layer as electron-selective contact $(\mathrm{p}(-)$ and $\mathrm{n}(-))$, the ZTGN composition does not affect 


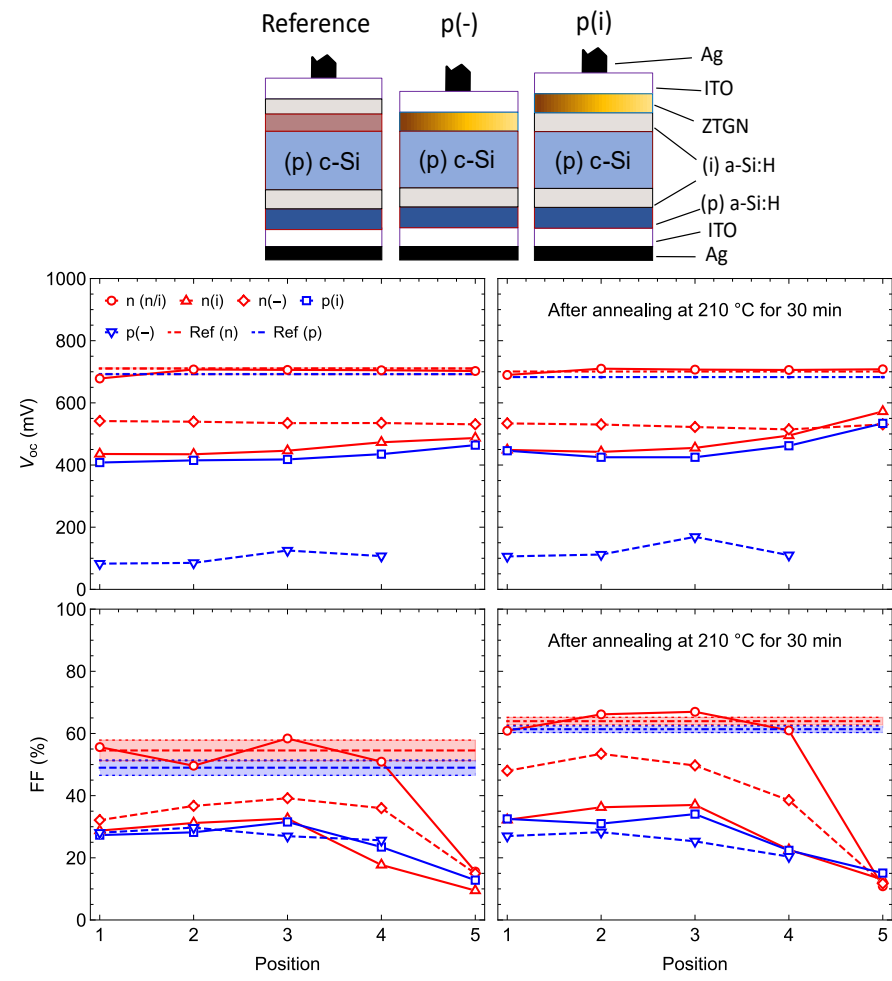

Fig. 5. $\mathrm{V}_{\mathrm{oc}}$ and FF at STC as a function of position and device architecture. Two architectures were added, using p-type wafers. For the Ge-rich cell on $\mathrm{p}$-Si, the IV curve showed a strong blocking behavior in the entire voltage range studied, and it was not possible to extract a reliable $\mathrm{V}_{\mathrm{oc}}$. Results from standard $\mathrm{p}$ and $\mathrm{n}$ SHJ solar cells are also shown for comparison. Blue and red bands denote the $95 \% \mathrm{CI}$ for the $\mathrm{V}_{\mathrm{oc}}$ (not visible) and FF from $10 \mathrm{SHJ}$ reference solar cells on $\mathrm{n}$ and $\mathrm{p}-\mathrm{Si}$.

$\mathrm{V}_{\mathrm{oc}}$, but the wafer type significantly influences it, suggesting that the wafer itself is contributing to the contact behavior [20]. The strong $\mathrm{V}_{\mathrm{oc}}$ difference between $\mathrm{p}(-)$ and $\mathrm{n}(-)$ devices is an indication of Fermi-level pinning at the c-Si surface, close to the (p) and (n) c-Si Fermi level, which suggests a defective interface.

For cells featuring an a-Si:H passivation layer, which enhances the role of the contact work function [27], the $\mathrm{V}_{\mathrm{oc}}$ values were similar on either wafer type due to a strong increase for cells on the $\mathrm{p}$-Si, but a slight decrease for cells on the $\mathrm{n}-\mathrm{Si}$. The presence of the (i)a-Si:H layer thus appears to efficiently suppress this quasi-Fermi-level pinning. The $\mathrm{V}_{\mathrm{oc}}$ values were nevertheless still similar for all compositions, with a $100 \mathrm{mV}$ increase towards positions 5. Since the $\mathrm{V}_{\mathrm{oc}}$ is determined by the quasi-Fermi level difference between the positive and negative contacts (and the hole contact stack is the same for all cells), this similar $\mathrm{V}_{\mathrm{oc}}$ regardless the wafer type means that the quasi-Fermi levels at the electron contact are essentially independent of composition, with a slight decrease in energy (i.e. moving towards vacuum energy) for samples with higher Ge content. This is consistent with the estimated work function values discussed in the previous section. Also, these $\mathrm{V}_{\mathrm{oc}}$ values are in accordance with the ones obtained by Bivour et al. in [27], when using metal with various workfunctions in direct contact to the (i)a-si:H layer.

Annealing had a mild influence on $\mathrm{V}_{\mathrm{oc}}$ for all compositions, except the Ge-rich one for which a close to $100 \mathrm{mV}$ increase was seen. This can be attributed to a recovery of sputtering damage, or an improvement of the electron-selectivity of the layer (e.g. decrease of work-function). The better performance in terms of $\mathrm{V}_{\mathrm{oc}}$ of the Ge-rich sample when the (i)a-Si:H layer is present does not correlate with the work function extracted from the JV-curve fittings presented in previous section. Specific care was taken to obtain accurate $V_{o c}$ values despite the flatness of the curve around open circuit and is deemed trustworthy. Compared to the simulation results, this indeed correlates to a lower leakage of holes from these fittings (lower $j_{0 p}^{Z T G N}$ ) which suggests improved passivation from this stack. This could be due to a lower defectivity of the layer, or to the fact that the valence-band edge is getting further away from the silicon one, preventing more efficiently hole recombination.

Samples including both an (i)a-Si:H and (n)a-Si:H layer under the ZTGN film showed similar $V_{o c}$ to the reference sample, confirming that selectivity and passivation are not affected in this case. Similar FF were also measured, except for the Ge-rich case. For this composition, as also observed on Fig. 3, a prominent barrier to charge extraction was still observed.

Turning to FF of samples not including the (n)a-Si:H layer, low values were observed. The n(-) samples performed slightly better than the others (as also seen in Fig. 3), which indicates that the too high work function (low selectivity) of ZTGN is unable to screen the barrier to charge extraction imposed by the a-Si:H layer, as was the case for solar cells with ZTN as electron contacts [12].

\section{Discussion}

As seen in Fig. 4, the Fermi-level of ZTGN showed only a slight decrease in energy (vacuum as reference) towards the Ge-rich sample. Upon increasing of the bandgap, the conduction band of ZTGN is therefore shifting upwards, leading to better alignment with c-si conduction band. However, since the changes in the Fermi level are much lower than the shift of the $\mathrm{CB}$, the carrier density in the Ge-rich ZTGN film remains too low to make it a good electron-selective contact, leading to a strong decrease in FF. Therefore, the Sn-rich layer is a low-bandgap conductive material, whereas Ge-rich films are higher-bandgap yet insulating.

Looking at sample $\mathrm{n}(-)$ in Fig. 5 corresponding to no a$\mathrm{Si}: \mathrm{H}$ layer between the c-Si wafer and the ZTGN layer, best FF is reached for position 2. From Fig. 4, this corresponds to a highly doped layer with a slight negative conductionband offset with crystalline silicon. Conversely, when a-Si:H is present (samples $n(i)$ and $n(n / i)$ ), best FF is reached for position 3, which corresponds to a slight positive conductionband offset with c-Si, and an approximate band alignment with a-Si:H. Deviating from these conditions leads to reduced FF, similarly to observations from [28] for hole-selective contacts.

To illustrate the effects of work function, doping and electron affinity on the band diagram of the n-Si/(i) a-Si:H/ZTGN, and therefore on the cell performance, simulations were carried out in PC1D considering a front-junction solar cell with three 


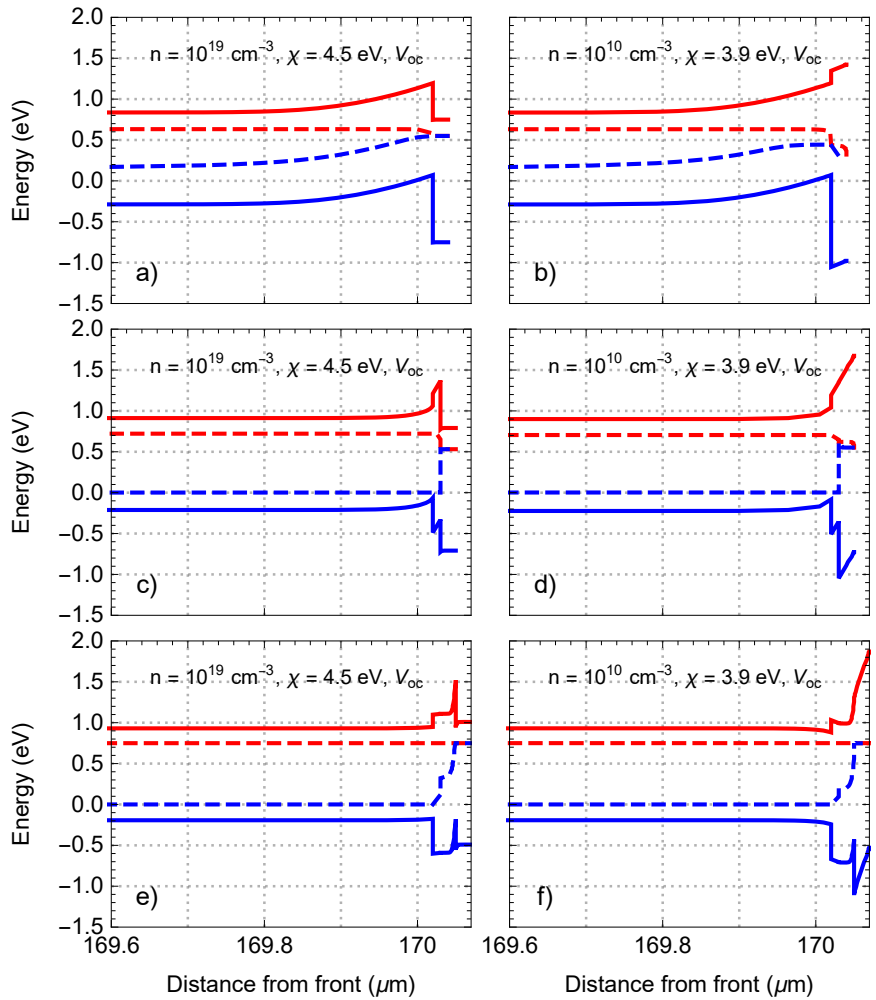

Fig. 6. PC1D simulations showing the band diagrams at $\mathrm{V}_{\mathrm{oc}}$ for a test layer with high extrinsic electron doping and electron affinity (left column), and with low doping and electron affinity (right column), for the interface between the test layer and n-Si (a, b), n-Si/(i) a-Si:H (c, d), and n-Si/(i) a-Si:H/(n) a-Si:H (e, f). Solid lines denote $\mathrm{CB}$ and VB positions, while dashed lines represent the quasi-Fermi levels.

regions: hole contact/n-Si/a-Si:H/Test layer, where the test layer was making the role of $\mathrm{Sn}$ and Ge-rich samples.

Two illustrative situations were considered: on the one hand an unfavorable electron affinity of $4.5 \mathrm{eV}$ but high doping of $1 \times 10^{19} \mathrm{~cm}^{-3}$, representative of the Sn-rich side, and on the other hand a suitable electron affinity of $3.9 \mathrm{eV}$ but low doping of $1 \times 10^{10} \mathrm{~cm}^{-3}$, representative of the Ge-rich side.

Fig. 6 shows the obtained band diagrams at open-circuit condition for these two cases, for either only the studied layer as contact, or with the addition of an (i) a-Si:H layer, or with an (i/n) a-Si:H layer stack. In Fig. 6a and b, the c-Si suffered a band bending due to the work function mismatch. Moreover, in the case of Fig. 6b, an additional barrier to transport is induced by the layer itself, independent of the bending in the c-Si.

Adding an (i) a-Si:H, most of the band bending is transferred to the a-Si:H layer. Due to the additional band offset, the barrier is even slightly higher yet narrower. In Fig. 6b, the resistive nature of the test layer, due to its poor doping, becomes even more prominent than in the previous case, which correlates well with the very low FF seen in Fig. 5. Inserting a doped (n) a-Si:H layer does not change the barrier height, but renders it much narrower due to the high doping in the (n) a-Si:H layer. The hole leakage towards the electron contact is also strongly decreased. This results in an increase in performance, as it was actually observed for architecture "n (n/i)" in Fig. 3. In Fig. 6e, the band diagram suggests an efficient electron-selective contact, which is what we observed. The appropriate work function of the (n) a-Si:H layer ensures electron selectivity, and its high doping screens efficiently the workfunction mismatch with the subsequent layer (mimicking here Sn-rich ZTGN, but the situation would be similar with an ITO layer). On the other hand, in Fig. 6f, although electron selectivity is similarly granted by the (n) a-Si:H layer, the subsequent layer still imposes a barrier due to its low doping, which cannot be screened. Strong resistive losses are thus expected in all architectures for the Ge-rich solar cell, which was observed through strong s-shapes.

Thus, these simulations point that the s-shapes seen in Fig. 3 are caused by the barriers for electron extraction at the interface c-Si/(i) a-Si:H/ZTGN as a result of a too high work function in both cases, and additionally by the ZTGN layer itself when its doping is low, as for the Ge-rich layer. Only the latter is expected when an (n) a-Si:H layer is inserted underneath the ZTGN film. Thus going one step further in the analysis, we can separate these two barriers as one being intrinsic to the contact selectivity, and the other one being external-thus being similar to e.g. the series resistance of the front metallic grid of the solar cell.

Considering that this is a non-Ohmic passive element in series, we can draw an equivalent circuit corresponding to the "n(n/i)", "n(i)" and "Reference" structures from Fig. 2. These are displayed on Fig. 7 a)-c), with the S-shape caused by the ZTGN layer itself being represented as a resistor with an S, and the changes in the contact selectivity being represented by a different diode color. Using these equivalent circuits, an obvious equation to determine the intrinsic $\mathrm{V}(\mathrm{J})$ characteristic of the contact is then:

$$
V_{\text {int }}(J)=V_{n(i)}(J)-\left(V_{n(n / i)}(J)-V_{\text {ref }}(J)\right)
$$

Normalizing each of the JV curves, it is therefore possible to isolate the JV characteristic of the S-shape element by subtracting "Reference" from the " $n(n / i)$ ". Removing this from the " $n(i)$ " JV curve renders the JV characteristic corresponding to the intrinsic performance of the contact.

These operations are shown in Fig. 7 d)-e) for the Gerich case (position 5), and the resulting JV characteristic is similar to the ones observed for the other four positions, as was expected from the similar value of the work function. Fitting this calculated characteristic with the previously-used exchange-current model results in $J_{0}$ values shown in Table 1 as "Position 5 - S". These are more consistent with the ones observed for Positions 1-4. Although these calculations are simplistic, and neglect e.g. the influence of the electrode, this confirms that the poor performance observed in position 5 can be split in two causes, with only one of them respecting the hypothesis of the exchange current density model, i.e. only one of them being an intrinsic contact selectivity issue.

\section{COnClusion}

This work reported the characterization of ZTGN layers on glass and the performance assessment of SHJ solar cells featuring these layers as electron-selective contacts. In the investigated composition range, bandgap was shown to be tunable 
a) Reference

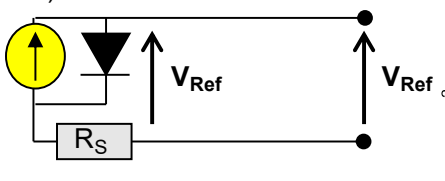

b) $n(n / i)$

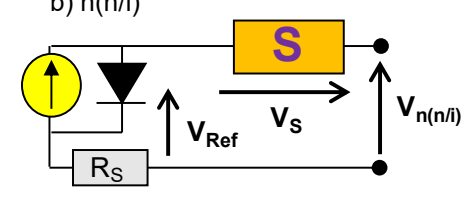

c) $\mathrm{n}(\mathrm{i})$

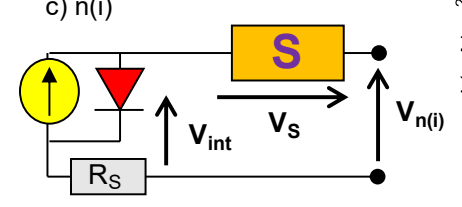

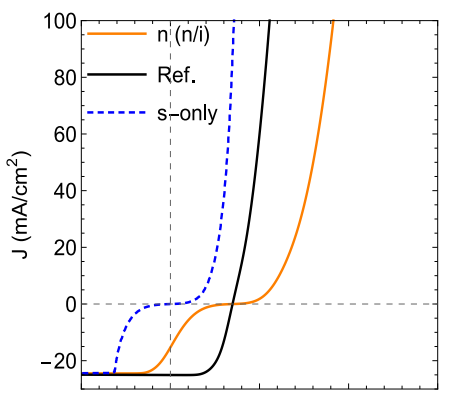

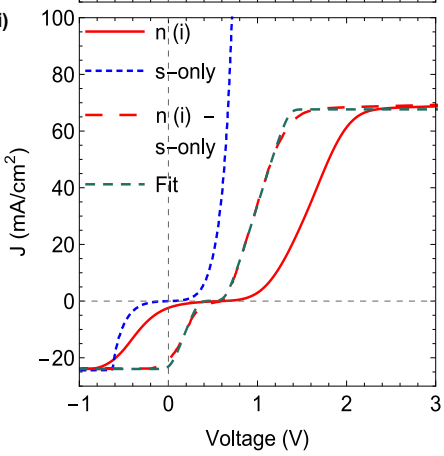

Fig. 7. Equivalent circuits corresponding to the reference cell (a), architectures "n (n/i)" (b) and "n (i)" (c). The s-shapes are represented by a resistor " $\mathrm{S}$ " in series, while the red diode represents a bad contact quality. The resulting JV curves from the operations given by (3) are shown in d) and e), where s-only is given by $V_{n(n / i)}(J)-V_{\text {ref }}(J)$. The light blue curve is the JV curve of the intrinsic performance of the contact with the Ge-rich ZTGN layer.

between $1.6 \mathrm{eV}$ and $2.3 \mathrm{eV}$ and conductivity and activation energy from $7 \mathrm{~S} \mathrm{~cm}^{-1}$ and $17 \mathrm{meV}$ to $2.5 \times 10^{-10} \mathrm{~S} \mathrm{~cm}^{-1}$ and $610 \mathrm{meV}$, respectively. Nevertheless, for all compositions, ZTGN performs similarly as electron-selective contact with low $\mathrm{V}_{\mathrm{oc}}$ (between $450 \mathrm{mV}$ and $580 \mathrm{mV}$ ) and FF.

Strong Fermi-level pinning at the c-Si interface was observed when the layer was in direct contact with the wafer, which was effectively suppressed when inserting an intrinsic a-Si:H layer.

Following an exchange-current modeling, the poor electronselective ability of these layers can be attributed to the ZTGN work function being around $4.3 \mathrm{eV}$, i.e. within the silicon bandgap, independently of the $\mathrm{Sn}$ and Ge contents. The Snrich composition is suggested to be limited by its too high electron affinity, whereas the Ge-rich composition combines a too low electron affinity with a too low doping (too high activation energy). This causes two different barriers for electron extraction, one being intrinsic to the selectivity of the contact and originating from unfavorable band bending in the silicon itself, and the other being external and due to the resistivity of the layer itself. Doping of Ge-rich ZTGN layers is therefore suggested as a required next step.

\section{ACKNOWLEDGMENT}

The authors thank Sylvain Dunand, Cedric Bucher and Nicolas Furst (EPFL PV-Lab) for technical support with some equipment. D. F. thankfully acknowledges the National Council for Scientific and Technological Development CNPq (Brazil) and the Federal Commission for Scholarships (Switzerland) for the grants received, essential for the development of this work. M.B. acknowledges Angela N. Fioretti

for sparking his interest in the $\mathrm{Zn}-\mathrm{IV}-\mathrm{N}_{2}$ material family and introducing him to the beauty of combinatorial analysis.

\section{REFERENCES}

[1] I. S. Khan, K. N. Heinselman, and A. Zakutayev, " Review of ZnSnN 2 semiconductor material ," Journal of Physics: Energy, vol. 2, no. 3, p. 032007, 2020 .

[2] F. Deng, H. Cao, L. Liang, J. Li, J. Gao, H. Zhang, R. Qin, and C. Liu, "Determination of the basic optical parameters of $\mathrm{ZnSnN}_{2}$," Optics Letters, vol. 40, no. 7, p. 1282, 2015.

[3] C. L. Melamed, J. Pan, A. Mis, K. Heinselman, R. R. Schnepf, R. Woods-Robinson, J. J. Cordell, S. Lany, E. S. Toberer, and A. C. Tamboli, "Combinatorial investigation of structural and optical properties of cation-disordered ZnGeN2," Journal of Materials Chemistry C, vol. 8, no. 26, pp. 8736-8746, 2020.

[4] A. D. Martinez, A. N. Fioretti, E. S. Toberer, and A. C. Tamboli, "Synthesis, structure, and optoelectronic properties of II-IV-V2 materials," Journal of Materials Chemistry A, vol. 5, no. 23, pp. 11418-11435, 2017.

[5] N. C. Coronel, L. Lahourcade, K. T. Delaney, A. M. Shing, and H. A. Atwater, "Earth-abundant $\mathrm{ZnSnxGe1-xN2}$ alloys as potential photovoltaic absorber materials," in 2012 38th IEEE Photovoltaic Specialists Conference. IEEE, 6 2012, pp. 003 204-003 207. [Online]. Available: http://ieeexplore.ieee.org/document/6318259/

[6] P. Narang, S. Chen, N. C. Coronel, S. Gul, J. Yano, L. W. Wang, N. S. Lewis, and H. A. Atwater, "Bandgap tunability in $\mathrm{Zn}(\mathrm{Sn}, \mathrm{Ge}) \mathrm{N} 2$ semiconductor alloys," Advanced Materials, vol. 26, no. 8, pp. 12351241, 2014.

[7] N. Beddelem, S. Bruyère, F. Cleymand, S. Diliberto, C. Longeaud, S. 1. Gall, R. Templier, P. Miska, and B. Hyot, "Structural and functional properties of $\mathrm{Zn}(\mathrm{Ge}, \mathrm{Sn}) \mathrm{N} 2$ thin films deposited by reactive sputtering," Thin Solid Films, vol. 709, no. October 2019, p. 138192, 2020. [Online]. Available: https://doi.org/10.1016/j.tsf.2020.138192

[8] A. M. Shing, N. C. Coronel, N. S. Lewis, and H. A. Atwater, "Semiconducting $\mathrm{ZnSnxGe1-xN2}$ alloys prepared by reactive radiofrequency sputtering," APL Materials, vol. 3, no. 7, 2015. [Online]. Available: http://dx.doi.org/10.1063/1.4927009

[9] K. Javaid, W. Wu, J. Wang, J. Fang, H. Zhang, J. Gao, F. Zhuge, L. Liang, and H. Cao, "Band Offset Engineering in ZnSnN2-Based Heterojunction for Low-Cost Solar Cells," ACS Photonics, vol. 5, no. 6, pp. 2094-2099, 2018.

[10] K. Javaid, J. Yu, W. Wu, J. Wang, H. Zhang, J. Gao, F. Zhuge, L. Liang, and H. Cao, "Thin Film Solar Cell Based on ZnSnN2/SnO Heterojunction," Physica Status Solidi - Rapid Research Letters, vol. 12 , no. 1, pp. 1-7, 2018.

[11] R. Qin, H. Cao, L. Liang, Y. Xie, F. Zhuge, H. Zhang, J. Gao, K. Javaid, C. Liu, and W. Sun, "Semiconducting ZnSnN2 thin films for Si/ZnSnN2 p-n junctions," Applied Physics Letters, vol. 108, no. 14, 2016. [Online]. Available: http://dx.doi.org/10.1063/1.4945728

[12] A. N. Fioretti, M. Boccard, A. C. Tamboli, A. Zakutayev, and C. Ballif, "Nitride layer screening as carrier-selective contacts for silicon heterojunction solar cells," in AIP Conference Proceedings, vol. 1999, 2018, p. 040007. [Online]. Available: http://aip.scitation.org/doi/abs/10.1063/1.5049270

[13] R. V. Chavali, S. De Wolf, and M. A. Alam, "Device physics underlying silicon heterojunction and passivating-contact solar cells: A topical review," Progress in Photovoltaics: Research and Applications, vol. 26, no. 4, pp. 241-260, 2018.

[14] Y. Liu, Y. Li, Y. Wu, G. Yang, L. Mazzarella, P. Procel-Moya, A. C. Tamboli, K. Weber, M. Boccard, O. Isabella, X. Yang, and B. Sun, "High-Efficiency Silicon Heterojunction Solar Cells: Materials, Devices and Applications," Materials Science and Engineering R: Reports, vol. 142, no. July, 2020.

[15] R. V. K. Chavali, J. V. Li, C. Battaglia, S. De Wolf, J. L. Gray, and M. A. Alam, "A generalized theory explains the anomalous suns- V OC response of si heterojunction solar cells," IEEE Journal of Photovoltaics, vol. 7, no. 1, pp. 169-176, 2017.

[16] E. T. Roe, K. E. Egelhofer, and M. C. Lonergan, "Exchange current density model for the contact-determined current-voltage behavior of solar cells," Journal of Applied Physics, vol. 125, no. 22, 2019.

[17] W. Lin, M. Boccard, S. Zhong, V. Paratte, Q. Jeangros, L. Antognini, J. Dréon, J. Cattin, J. Thomet, Z. Liu, Z. Chen, Z. Liang, P. Gao, H. Shen, and C. Ballif, "Degradation Mechanism and Stability Improvement of Dopant-Free $\mathrm{ZnO} / \mathrm{LiF} \times \mathrm{Al}$ Electron Nanocontacts in Silicon Heterojunction Solar Cells," ACS Applied 
Nano Materials, p. acsanm.0c02475, 2020. [Online]. Available: https://pubs.acs.org/doi/10.1021/acsanm.0c02475

[18] J. Brest, S. Greiner, B. Boskovic, M. Mernik, and V. Zumer, "SelfAdapting Control Parameters in Differential Evolution: A Comparative Study on Numerical Benchmark Problems," IEEE Transactions on Evolutionary Computation, vol. 10, no. 6, pp. 646-657, 122006. [Online]. Available: https://ieeexplore.ieee.org/document/4016057/

[19] D. Fébba, E. Bortoni, A. Oliveira, and R. Rubinger, "The effects of noises on metaheuristic algorithms applied to the PV parameter extraction problem," Solar Energy, vol. 201, no. November 2019, pp. 420-436, 5 2020. [Online]. Available: https://doi.org/10.1016/j.solener.2020.02.093 https://linkinghub.elsevier.com/retrieve/pii/S0038092X20302206

[20] A. Onno, C. Chen, P. Koswatta, M. Boccard, and Z. C. Holman, "Passivation, conductivity, and selectivity in solar cell contacts: Concepts and simulations based on a unified partial-resistances framework," Journal of Applied Physics, vol. 126, no. 18, 2019.

[21] E. T. Roe, K. E. Egelhofer, and M. C. Lonergan, "Limits of Contact Selectivity/Recombination on the Open-Circuit Voltage of a Photovoltaic," ACS Applied Energy Materials, vol. 1, no. 3, pp. 1037-1046, 2018.

[22] J. Dreon, J. Cattin, G. Christmann, D. Fébba, V. Paratte, L. Antognini, W. Lin, S. Nicolay, C. Ballif, and M. Boccard, "Performance Limitations and Analysis of Silicon Heterojunction Solar Cells Using Ultra-Thin MoO\$_\{ $\backslash \mathrm{rm}$ x $\} \$$ Hole-Selective Contacts," IEEE Journal of Photovoltaics, pp. 1-9, 2021. [Online]. Available: https://ieeexplore.ieee.org/document/9453050/

[23] W. Walukiewicz, J. W. Ager, K. M. Yu, Z. Liliental-Weber, J. Wu, S. X. Li, R. E. Jones, and J. D. Denlinger, "Structure and electronic properties of InN and In-rich group III-nitride alloys," Journal of Physics D: Applied Physics, vol. 39, no. 5, 2006.

[24] C. G. Van de Walle and J. Neugebauer, "Universal alignment of hydrogen levels in semiconductors, insulators and solutions," Nature, vol. 423, no. 6940, pp. 626-628, 6 2003. [Online]. Available: http://www.nature.com/articles/nature01665

[25] X. Yang, K. Weber, Z. Hameiri, and S. De Wolf, "Industrially feasible, dopant-free, carrier-selective contacts for high-efficiency silicon solar cells," Progress in Photovoltaics: Research and Applications, vol. 25, no. 11, pp. 896-904, 11 2017. [Online]. Available: http://doi.wiley.com/10.1002/pip.2901

[26] S. Zhong, M. Morales-Masis, M. Mews, L. Korte, Q. Jeangros, W. Wu, M. Boccard, and C. Ballif, "Exploring co-sputtering of $\mathrm{ZnO}: \mathrm{Al}$ and $\mathrm{SiO} 2$ for efficient electron-selective contacts on silicon solar cells," Solar Energy Materials and Solar Cells, vol. 194, no. November 2018, pp. 67-73, 2019. [Online]. Available: https://doi.org/10.1016/j.solmat.2019.02.005

[27] M. Bivour, C. Reichel, M. Hermle, and S. W. Glunz, "Improving the a-Si:H(p) rear emitter contact of n-type silicon solar cells," Solar Energy Materials and Solar Cells, vol. 106, pp. 11-16, 2012. [Online]. Available: http://dx.doi.org/10.1016/j.solmat.2012.06.036

[28] R. Woods-Robinson, A. N. Fioretti, J. Haschke, M. Boccard, K. A Persson, and C. Ballif, "Evaluating Materials Design Parameters of Hole-Selective Contacts for Silicon Heterojunction Solar Cells," IEEE Journal of Photovoltaics, pp. 1-12, 2020. 